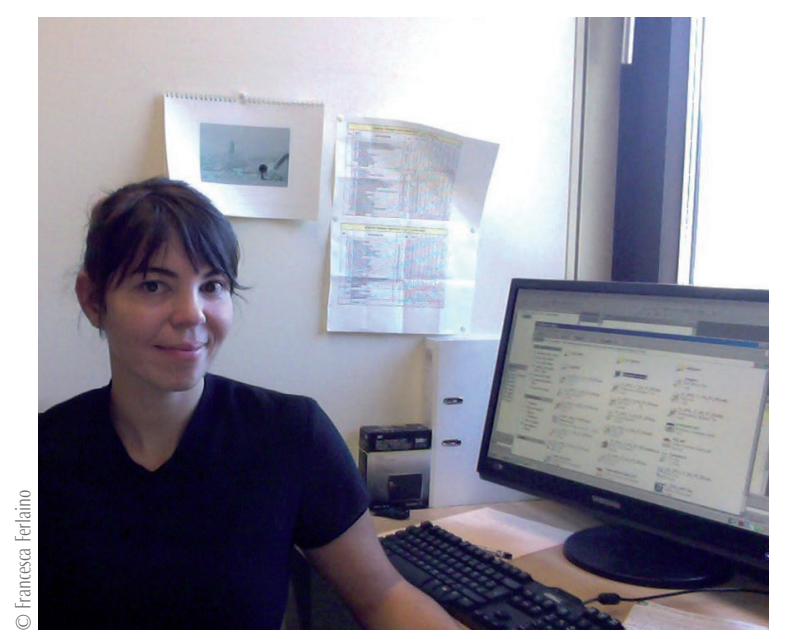

\title{
Une jeune physicienne européenne qui réussit : Francesca Ferlaino
}

Bonjour Francesca, nous sommes aujourd'hui ensemble à Washington à la très prestigieuse conférence internationale de physique atomique ICAP 2014, à laquelle assistent 900 personnes. Sur les 49 orateurs invités, on compte seulement 5 femmes et tu es l'une d'entre elles. Peux-tu retracer brièvement les grandes lignes de ta brillante carrière qui te valent ce beau succès à l'âge de 36 ans ? Après des études à Naples où je suis née, puis à Trieste, j'ai fait une thèse à Florence, prolongée par deux ans de postdoc sur place dans le domaine des atomes froids au Laboratoire européen de spectroscopie non linéaire (LENS). Puis je suis allée à Innsbruck pour un second postdoc dans un excellent laboratoire du domaine, où je suis finalement restée jusqu'à aujourd'hui. Mes recherches sur les mélanges fermions/bosons dans les réseaux optiques, puis sur la condensation de l'atome d'erbium (mon sujet personnel) ont très bien marché. J'ai bénéficié de bourses européennes et reçu un certain nombre de prix qui m'ont permis de développer mes projets. Je suis lauréate d'un contrat ERC junior et d'un grand prix autrichien, et maintenant d'un prix de la fondation Humboldt de $5 \mathrm{M} €$ pour aller à Ulm comme professeur (ce que je viens de refuser, car on m'offre presqu'autant après négociation pour rester à Innsbruck). Je suis donc maintenant professeur titulaire de mon poste à l'université et je dirige ma propre équipe de recherche en toute indépendance, autour d'expériences très complexes qui affrontent une compétition internationale très forte.

Comment s'est déterminée ta vocation pour la recherche, pourquoi as-tu choisi la physique, qu'est-ce qui a motivé ta mobilité géographique, as-tu été aidée ou encouragée?

À Naples, pendant ma scolarité, j'ai subi un mauvais enseignement de physique et j'ai fait des études classiques. Toutefois, j'ai gardé un souvenir marquant d'un excellent professeur de math en classe de $4^{\mathrm{e}}$; j'ai aussi été marquée par la visite passionnante d'une centrale nucléaire en France ; de plus, un ami plutôt original de ma famille m'a encouragée à prendre la physique à l'université. En fait, mes parents auraient souhaité une autre carrière pour moi, médecine par exemple : ils trouvaient à juste titre la physique comme trop risquée... C'est pourquoi j'ai préféré quitter Naples très jeune pour aller à Florence, c'était le choix de la liberté. Je dois ajouter que j'ai été très influencée par des modèles féminins. C'est d'ailleurs en France que j'ai rencontrée quelques femmes juste un peu plus âgées que moi, comme Hélène Perrin au Laboratoire de physique des lasers à Villetaneuse, dont la réussite en recherche et dans la vie personnelle était évidente : cela m'a énormément encouragée.
Tu as choisi de t'établir à Innsbruck, dans un pays où, comme en Allemagne, on ne favorise guère le travail des femmes. Tu es de plus une jeune femme pleine de féminité, jolie et aussi très élégante. Comment s'est passée ton arrivée dans un milieu presque totalement masculin ? Ressens-tu encore la pression des stéréotypes et comment y réagis-tu?

J'ai eu un choc en arrivant en Autriche, après mon passage à Florence où ma codirectrice de thèse était une femme et où il y avait la mixité complète dans le laboratoire. Je n'ai pas souffert directement de misogynie flagrante, mais plutôt j'ai parfois remarqué une attitude généralisée des hommes enclins à ignorer les femmes. J'ai eu mille occasions de noter des comportements qui ne sont pas toujours "politiquement corrects ». Par exemple, dans un groupe l'homme qui répond à une question que j'ai posée évite de me regarder en face. Cela donne l'impression d'être transparente et je ne sais pas trop comment interpréter ce comportement. En tout cas, j'ai décidé de ne pas m'en sentir affectée et plutôt d'en rire : j'ai pris le parti d'ignorer d'être ignorée !

Je voudrais aussi ajouter que les stéréotypes cessent très rapidement quand les gens me connaissent. À ce point, c'est le rapport direct qui dicte les règles comportementales.

Pour ce qui est de la féminité, je fais attention à ne pas provoquer, je m'habille simplement au travail, je prends garde de maintenir fermement un cercle d'isolation autour de moi et je n'ai pas de problèmes.

$\mathrm{Tu}$ as maintenant deux très jeunes enfants (4 ans et 1 an). Considères-tu que tu réussis à maintenir un équilibre satisfaisant entre ta famille et ton travail de directrice d'une grande équipe ? Comment fais-tu?

J'ai la chance d'avoir une "nounou " qui vient de Naples et s'occupe de mes enfants. Évidemment cela coûte cher, mais c'est l'idéal dans ce pays (l'Autriche) où il y a très peu de crèches qui, de plus, n'acceptent pas les enfants de moins de 15 mois ! On commence cependant à trouver des jeunes filles au pair, mais ceux de mes collègues qui ont des moyens limités ont beaucoup de problèmes. Il n'y a pas d'aide de la société pour les femmes qui travaillent avec des enfants en bas âge ; par contre, on peut garder $80 \%$ de son salaire à rester chez soi pendant un an. C'est la mentalité en Autriche, vous avez de la chance en France.

Je considère aussi comme très importante la qualité de mes relations avec mon compagnon, le père de mes enfants, qui est éducateur social. Il a plus de temps que moi à passer à la maison et il est pour moi une aide énorme. Le choix du compagnon est fondamental. 


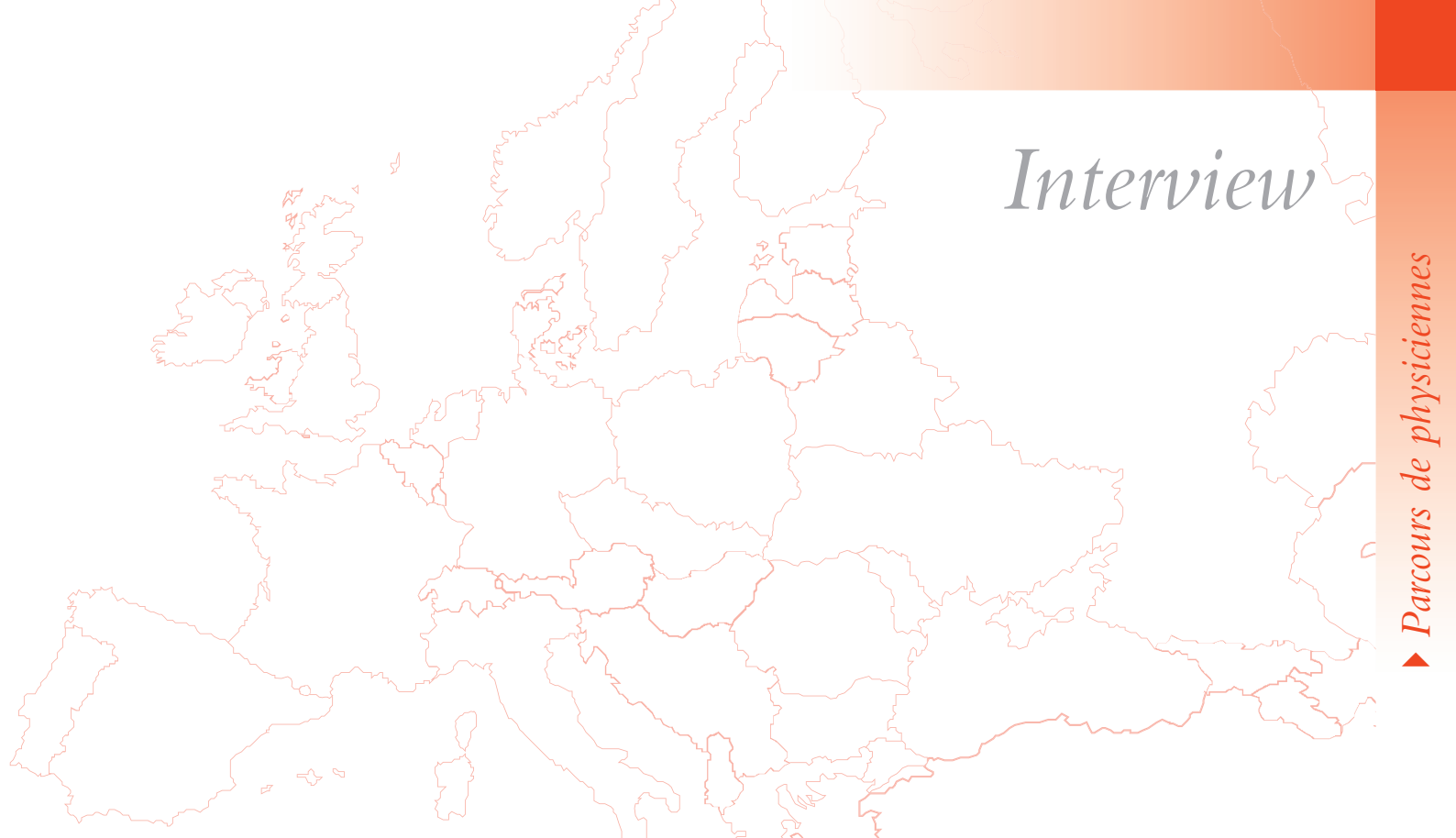

$\mathrm{Tu}$ es à la tête d'une équipe masculine de six personnes. Comment t'y prends-tu pour créer une bonne ambiance de travail ? Penses-tu que les femmes ont des atouts particuliers pour y parvenir? Je crois qu'il y a une bonne harmonie dans mon équipe. Mes contacts sont très forts avec mes doctorants et postdoctorants. Je m'applique à détecter les problèmes qu'ils peuvent avoir entre eux, ou avec moi, bien avant qu'ils apparaissent. Il faut à tout prix éviter les conflits et la guerre dans un labo! On peut toujours trouver une solution ; j'observe beaucoup ce qui se passe et je mets mon intuition dite féminine à contribution. À cet égard, je pense que les femmes ont plus de qualités que les hommes et qu'il faut qu'elles les mettent à profit.

De toute évidence, tu es une femme scientifique dont la réussite professionnelle est exceptionnelle et qui de plus est heureuse dans la vie. Tu es devenue à ton tour une femme modèle pour beaucoup d'autres jeunes femmes ; quels conseils leur donnerais-tu ? Comment vois-tu la solidarité entre femmes scientifiques et te considères-tu comme une militante féministe ?

En effet, beaucoup de femmes viennent me voir, souvent pour des conseils pratiques, ou juste pour être encouragées. Nous avons dans mon institut de sympathiques déjeuners réguliers de femmes en physique. Je leur dis qu'il ne faut pas avoir peur et surtout ne pas se sous-estimer. Pourtant, je ne me vois pas comme une militante féministe qui revendiquerait avec agressivité des droits particuliers pour les femmes dans ma profession. Je suis contre la lutte des classes entre les hommes et les femmes ! En réalité, ce que je crois important c'est d'obtenir des petites avancées concrètes qui facilitent la vie des femmes qui travaillent, de toutes les femmes d'ailleurs et pas seulement les scientifiques. Il faut des aides financières pour la maternité et le retour au travail au bout de quelques mois. Moi, je me bats pour les crèches. C'est toute la mentalité de la société que je voudrais voir changer !

Qu'aimerais-tu ajouter pour les lectrices de Reflets de la physique? Je suis préoccupée par la vision qu'ont les femmes des autres femmes. Souvent, dès qu'elles acquièrent un peu de pouvoir, elles se comportent comme les hommes vis-à-vis de leurs collègues femmes. En fait, elles intègrent les stéréotypes qui imprègnent leur milieu et font preuve sans s'en rendre compte des mêmes préjugés. Ce qui me tourmente, c'est que je me surprends moi-même parfois dans cet état d'esprit. Il faut être extrêmement vigilant vis-à-vis de soi-même. Appartenir à un groupe de femmes qui réfléchissent ensemble (comme Femmes \& Sciences en France) serait très utile à cet égard, mais cela n'existe pas encore à Innsbruck.

Pour terminer, pourrais-tu expliquer en termes simples en quoi consistent tes recherches?

Ma thèse à Florence a suivi de quelques années la découverte de la condensation de Bose-Einstein du sodium et du rubidium. J'ai pu étendre pour la première fois ces méthodes au potassium et produire un gaz ultra-froid de fermions dans un réseau optique, ouvrant la voie à des simulations quantiques de problèmes complexes en physique de la matière condensée. J'ai étudié les interactions entre espèces différentes, que j'ai pu faire varier entre zéro et quasiment l'infini à l'aide de champs magnétiques via des résonances dites de Feshbach. Plus récemment, j'ai abordé la physique des interactions à plusieurs corps en produisant transitoirement des particules liées composées de trois atomes de césium, nommées états d'Efimov. Mon sujet actuel concerne l'erbium, un atome de terre rare dont j'ai réussi pour la première fois la condensation de Bose-Einstein en 2012. J'exploite la physique de cet atome qui est très " riche", car il a beaucoup d'isotopes fermioniques et qu'il possède un grand moment dipolaire qui lui permet des interactions anisotropes à longue portée.

Interview réalisée le 8 août 2014 par Michèle Leduc

\section{Parcours}

Francesca Ferlaino a 36 ans - (Francesca.Ferlaino@uibk.ac.at)

2000 : D Diplôme de physique à l'Université Federico II (Naples, Italie).

2004 : $\quad$ Diplôme de thèse $(\mathrm{PhD})$ à l'Université de Florence sur le sujet :"Atomic Fermi gases in optical lattices (experiment)"

2004-2006 : Assistante de recherches au LENS (Florence, Italie)

2007-2011 : Postdoc puis professeur associé à l'Université d'Innsbruck (Autriche)

Depuis 2012 : Professeur titulaire à l'Université d'Innsbruck

Depuis 2013 : Directrice du Centre de Recherches "Physics-Innsbruck» 